\title{
e-VLBI with LOFAR
}

\section{Olaf Wucknitz ${ }^{* \dagger}$}

Argelander-Institut für Astronomie, Auf dem Hügel 71, 53121 Bonn, Germany

E-mail: wucknitz@astro.uni-bonn.de

LOFAR for the first time offers the possibility to image the radio sky at wavelengths of a few metres with a resolution in the arcsec range or better. This is made possible by international stations that provide baselines of several hundred $\mathrm{km}$ and turn LOFAR into an e-VLBI array. In this talk I give an overview of first high-resolution observations using the International LOFAR Telescope and discuss the particular challenges of long-baseline LOFAR observations.

In addition the first resolved LOFAR images of the Sun are presented.

10th European VLBI Network Symposium and EVN Users Meeting: VLBI and the new generation of radio arrays

September 20-24, 2010

Manchester Uk

* Speaker.

$\dagger$ This work is supported by the Emmy-Noether-Programme of the 'Deutsche Forschungsgemeinschaft', reference WU 588/1-1, and by a Marie Curie European Reintegration Grant within the 7th European Community Framework Programme, Contract No. PERG02-GA-2007-224897 'WIDEMAP'. 


\section{Introduction}

The Low Frequency Array (LOFAR) will soon consist of $\sim 40$ stations in the Netherlands plus international ones in Germany, France, England, Sweden, Poland, Finnland and hopefully also Italy, Spain and elsewhere. Currently all available international stations are in Germany: Effelsberg near Bonn, Tautenburg near Jena, Unterweilenbach near Munich and Bornim near Potsdam (see Fig. 1). Potsdam was not connected yet and could not be used for the work presented here. The baseline lengths between the Dutch core and German stations are about $300 \mathrm{~km}$ (Effelsberg), $400 \mathrm{~km}$ (Tautenburg) and $600 \mathrm{~km}$ (Unterweilenbach).

LOFAR will offer wide fields of view, observations with several independent beams and a high survey speed. Two subsystems cover a frequency range between about 10 and $250 \mathrm{MHz}$; Low Band Antennas (LBA) for 30(10)-80 MHz, and High Band Antennas (HBA) for 110-250 MHz.

On long baselines, sub-arcsec resolution will be possible even in the LBA band, which opens an entirely new parameter space for radio observations. Because of the high number of stations, full synthesis imaging is possible.
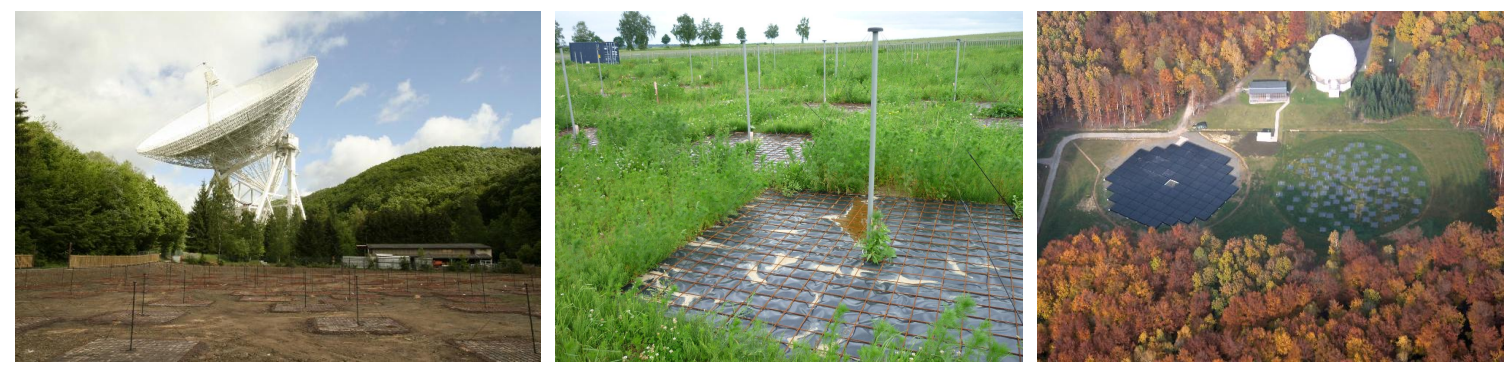

Figure 1: Left: LBA field of the Effelsberg station with the $100 \mathrm{~m}$ radio telescope in the background, centre: LBA antennas in Unterweilenbach, right: the Tautenburg station with HBA and LBA fields and the dome of the $2 \mathrm{~m}$ optical telescope.

\section{Long baseline issues}

A major issue on long baselines is the fact that the majority of sources (including calibrators) will at least be partially resolved so that the signal weakens considerably. In order to achieve a sufficient signal-to-noise ratio $(S / N)$ for imaging and calibration, the data have to be averaged in time and frequency. For this the dependence of phases on time and frequency has to be taken into account. Delays (proportional to derivatives of phase with respect to frequency) and rates (derivatives of phase with respect to time) have to be fitted and corrected for. In Very Long Baseline Interferometry (VLBI), this technique is known as fringe-fitting and is applied on a regular basis.

In the context of LOFAR, with the very low frequencies and very large fractional bandwidth, the procedure is considerably more complicated than in standard VLBI observations, because one has to distinguish between non-dispersive and dispersive delays. Non-dispersive delays ( $\tau$ independent of $v$ ) are due to clock offsets and errors in positions of stations and sources, while dispersive delays $\left(\tau \propto v^{-2}\right)$ are caused by the ionosphere. Generally it turns out that both are important. We find that the clock offsets can be much larger than the ionospheric delays (sometimes many $\mu$ sec when the clock synchronisation fails), but they tend to be constant with time and are thus easier to 
correct. Ionospheric delays of several $100 \mathrm{nsec}$ are often observed on long baselines at frequencies around $50 \mathrm{MHz}$. Both effects have to be taken into account when averaging over several subbands.

At the moment, fringe-fitting is only partly implemented in own software. I can fit both kinds of delays on individual baselines. Because the dispersion is not significant over a single subband, FFTs can be used within the subbands. Interpolated results of that are combined coherently over all subbands to determine multi-band delay solutions.

With the wide bands, the rates have to be treated as delay rates instead of phase rates, because the latter would be frequency dependent both for dispersive and non-dispersive delays. The software thus fits four parameters (plus a phase) for blocks of data that typically extend over 10-60 sec in time and up to $48 \mathrm{MHz}$ in frequency.

In addition it is found, not unexpectedly, that differential Faraday rotation between the stations is generally significant at lower frequencies and on long baselines. This means that the XX and YY correlations are not sufficient to determine Stokes I, because parts of the signal are shifted to the mixed correlations XY and YX. Finally the imaging on long baselines is much more difficult than for the Dutch array alone, because the $u v$ coverage is less complete.
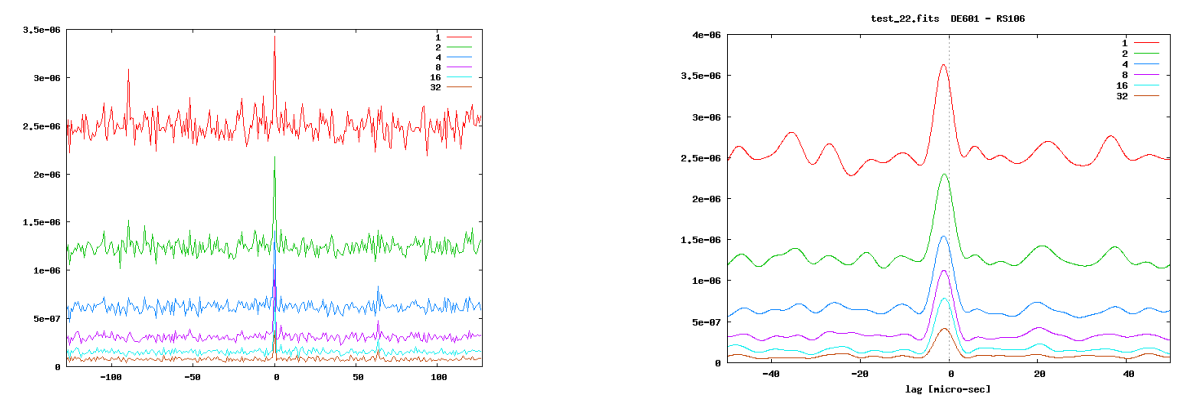

Figure 2: Delay spectra on a baseline between the Netherlands and Effelsberg. The different colours denote different coherent averaging times (labels in units of the integration time of $5 \mathrm{sec}$ ). The second plot with higher delay resolution shows that the peak is slightly offset from 0 .

\section{First long-baseline fringes}

Shortly after others had seen hints of short periods of consistent phases on LBA observations of 3C196 that included the station Effelsberg, I started a more systematic search for fringes in the parameter space of delay and rate. For the first attempt in August 2009, data of one subband were coherently averaged over short intervals, then Fourier transformed on the frequency axis to obtain delay spectra, and the power was averaged (incoherently) over one hour to increase the $S / N$. Results are shown in Fig. 2.

The clear peak in the delay spectra near a lag of 0 did not convince everybody at that time, because other effects may mimic real fringes caused by a sky signal, particularly if the peak is at exactly zero delay. Plotting the spectra with higher resolution revealed that the peak is in fact offset by about one $\mu$ sec.

A more systematic analysis followed in which the time-dependence was explicitly taken into account by scanning a two-dimensional (and finally four-dimensional) parameter space of delay 
and rate. See Fig. 3 for the first single-band and multi-band results, again for observations of 3C196.
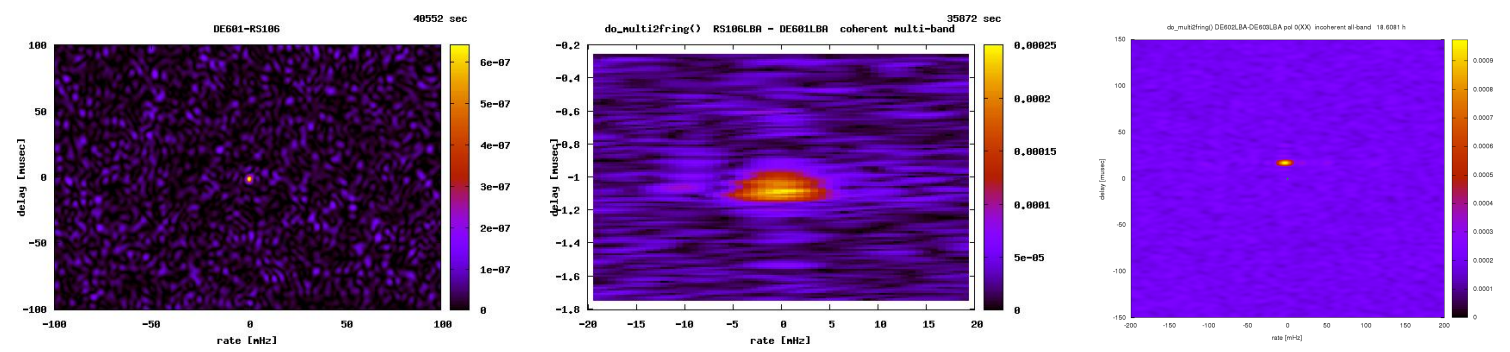

Figure 3: Fringe-rate/delay spectra for a short period of observations of 3C196 on long baselines. Left: single-band with low resolution on the vertical delay axis, baseline Netherlands-Effelsberg. Centre: coherent combination of many bands with much higher resolution in delay; same baseline. The strange shape of the peak is partly caused by not including dispersive effects in this plot. Right: First fringes on the baseline Tautenburg-Unterweilenbach. The delay of about $17 \mu \mathrm{sec}$ is caused by a clock offset in the Tautenburg station that resulted from an incorrect station position in the GPS configuration. This problem was fixed later.

This analysis started on the short baselines, where the strongest fringes were surprisingly found for delays and rates far from zero and of the same order of magnitude as the total geometric delays and rates. I found that the stronger fringes are resulting from other bright sources like Cygnus $\mathrm{A}$ or Cassiopeia A. Their signal, leaking in through high-order sidelobes of the station beam, can be stronger than the target signal even if they are more than $90^{\circ}$ away from the station beam centre.

\section{Results of fringe-analysis}

The most important result is the clear detection of fringes in long-baseline LBA observations. In addition a number of technical issues were found in the course of this analysis. Among them are the relatively large clock offsets in German stations, some confusion about the labelling of LBA polarisations under certain circumstances, a strong $8 \mathrm{MHz}$ ripple that resulted from accidentally disabling the cable-length compensation in the beam forming, the detection of strong differential Faraday rotation and an independent detection of a resonance near $63 \mathrm{MHz}$.

Most of these problems have been fixed later. The independent long-baseline analysis contributed significantly to that process, even though this was not the primary intention of my efforts.

\section{First long-baseline LBA maps}

For the first serious long-baseline imaging attempt, a $6 \mathrm{~h}$ observation on the target $3 \mathrm{C} 196$ on 12/13th Feb 2010 (data set D2010_16704) was used. Five Dutch and the three German stations produced a wide range of baselines lengths. Only a fraction of the 160 subbands $(30-80 \mathrm{MHz}$ with small gaps) showed a strong signal because of the aforementioned $8 \mathrm{MHz}$ ripple. In the first attempts, only the good subbands were used, later the weaker ones were included, too.

In the analysis I first corrected for the $1 \mu \mathrm{sec}$ and $17 \mu \mathrm{sec}$ clock offsets in Effelsberg and Tautenburg, applied some flagging, then converted the XX/XY/YX/YY polarisation combinations 

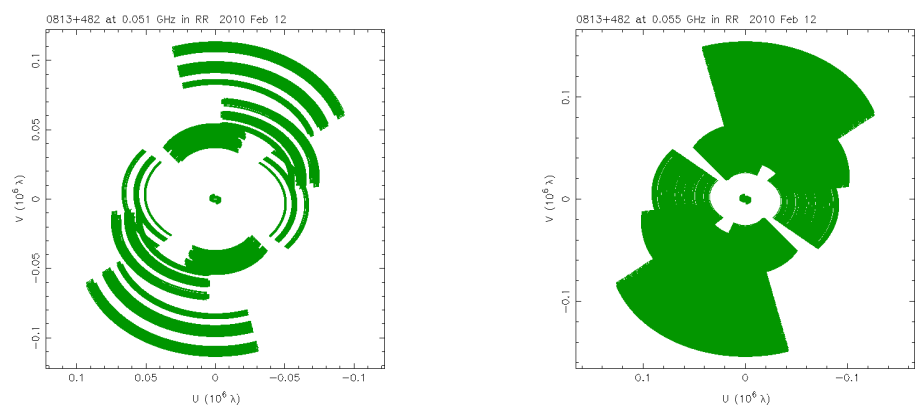

Figure 4: $u v$ coverage of the long-baseline LBA observations. Left: only the 'good' subbands, right: the entire frequency range. The Dutch baselines form the compact small region in the centre. All other baselines are combinations of/with German stations.
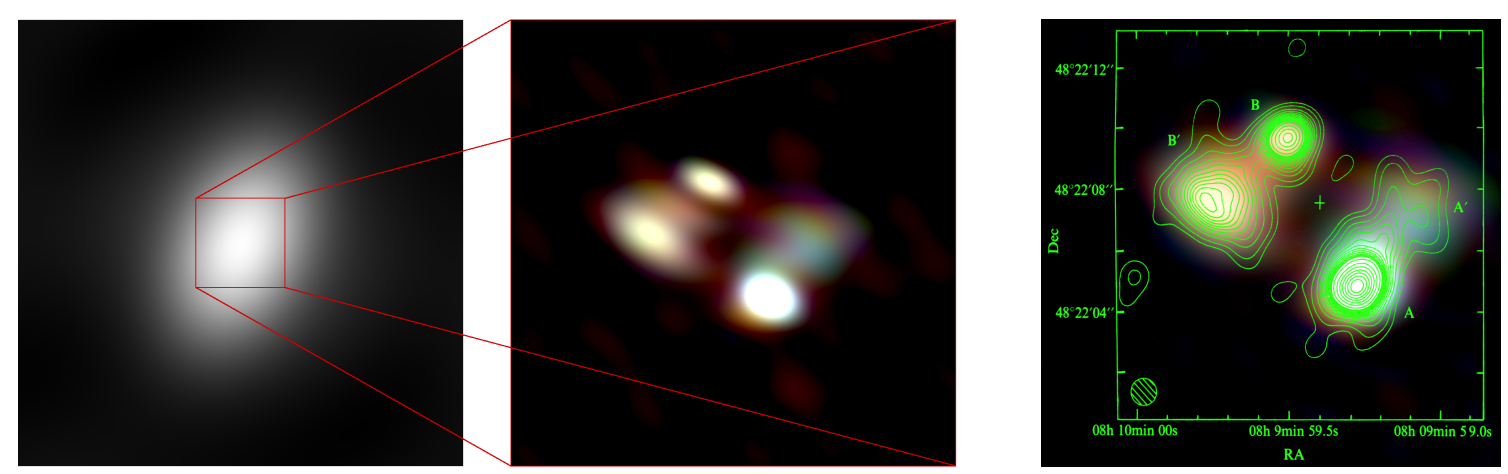

Figure 5: Left: Map of 3 C196 using only the Dutch baselines. The resolution of $35^{\prime \prime} \times 22^{\prime \prime}$ is not sufficient to resolve any details. Centre: Blowup using all the long baselines. With a resolution of $1 . " 5 \times 0$ "! 9 , the structures of the target are finally revealed. The colours are chosen to resemble what the human eye would see if it were sensitive to radiation at a wavelength ten million times larger than visible light. Right: Comparison with $408 \mathrm{MHz}$ MTRLI/MERLIN observations (contours from Lonsdale \& Morison [1]). The structures match surprisingly well, despite the factor of 8 in frequency. Note that the self-calibration process started conservatively with a point-source model and continued without prior knowledge of the $408 \mathrm{MHz}$ details.

to circular RR/RL/LR/LL in order to avoid differential Faraday rotation, averaged somewhat in time and frequency, and then finally imaged and self-calibrated RR and LL in AIPS and di fmap. For the final maps produced with difmap, Gaussian components with variable spectral indices were fitted to the data in order to correct the MFS (multi frequency synthesis) imaging for spectral index variations.

The $u v$ coverage is shown in Fig. 4. Images produced from these data are presented in Fig. 5.

\section{The Sun}

First interferometric observations of the Sun were tried on 9th June 2010 by the Solar KSP in Potsdam (PI: Gottfried Mann) with support from Bonn. My long-baseline software was used to produce pre-calibrated uvfits files that were imaged and self-calibrated in difmap. Fig. 6 compares the very first image of the Sun at $135 \mathrm{MHz}$ with other observations. The compact active region seen in that image varies on time-scales of seconds. In addition, dynamic spectra show 
significant variations with frequency. Both effects present a serious challenge for the calibration and imaging process.

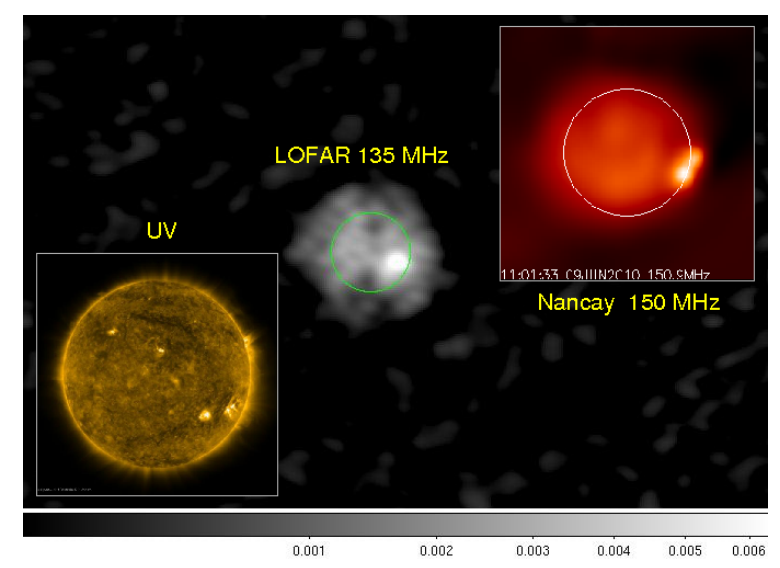

Figure 6: The very first resolved LOFAR image of the Sun compared with an image from the Nancay Radio Heliograph (right) and an uv image (left) of the same day. At LOFAR frequencies, the Sun is larger than in the optical. The presence of a compact active region is confirmed by the other observations.

\section{Summary}

This contribution proves that the long baselines of LOFAR actually work. They produce fringes, they can be calibrated, and they can be used to produce reliable high-resolution images of the low-frequency radio sky.

The highest-resolution image ever produced in the frequency range 30-80 MHz resulted from only three international (plus five Dutch) stations, a small part of the final array. Fringe-fitting methods are required to determine and correct dispersive and non-dispersive delays in order to allow averaging in frequency. Phase changes with time turn out to be not much worse on long baselines than on short ones. Beyond about $30 \mathrm{~km}$, the ionospheric patches are more or less independent of each other, so that variations do not increase with baseline length anymore. Even though the delays are larger, they can be calibrated because of their stability.

In order to analyse these data, new analysis techniques had to be developed and implemented. The results presented here were achieved with software written particularly for this purpose together with the standard packages AIPS and difmap.

First solar imaging attempts prove that LOFAR can resolve the Sun and show its strong variability as a function of time and frequency.

The original presentation of the conference talk (with a number of movies) is available from the author's homepage at http: //www. astro.uni-bonn. de/ wucknitz/publications / pub.php?2010_evn_lofar.

\section{References}

[1] Lonsdale, C. J. \& Morison, I.: 1980, Rotationally symmetric structure in two extragalactic radio sources, Nature 288, 66-69. 